\title{
ACKNOWLEDGMENT
}

We thank the reviewers listed below with the number of manuscripts assessed, by revisions performed during the year 2011. Thanks to this volunteer work, the Brazilian Journal of Cardiovascular Surgery (BJCVS) has published high quality articles, always assessed within strict criteria and the most absolute scientific accuracy, enabling our first Impact Factor was extremely positive.

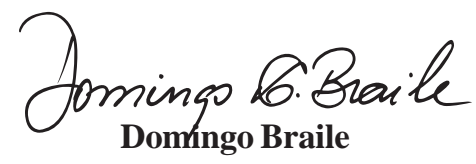

BJCVS Editor

\section{REVIEWERS 2011}

\begin{tabular}{|c|c|}
\hline Alexandre C Hueb ............... & Fernando Ribeiro de Moraes Neto ....... \\
\hline 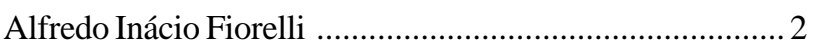 & 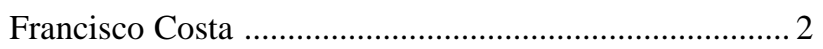 \\
\hline 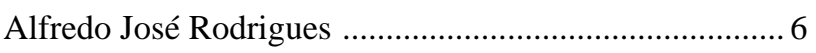 & 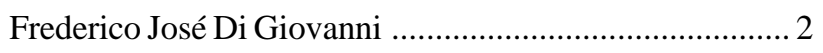 \\
\hline Ana Maria Rocha Pinto e Silva ...................................... 3 & Frederico Pires de Vasconcelos Silva ............................. 1 \\
\hline \multicolumn{2}{|l|}{ Antoninho Sanfins Arnoni ............................................ 1} \\
\hline Bruno Botelho Pinheiro . & Gilberto Venossi Barbosa ….............................................. 5 \\
\hline 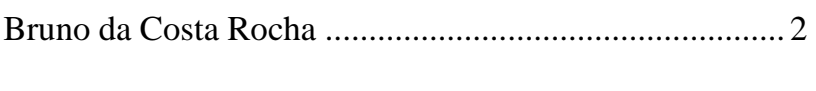 & Glaucia Basso ........................................................ 1 \\
\hline 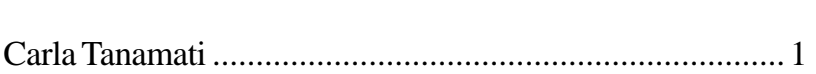 & 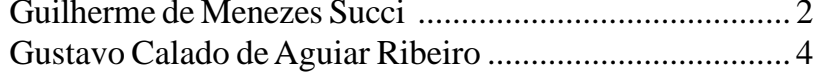 \\
\hline 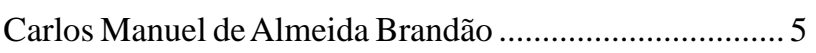 & Gustavo Glotz de Lima \\
\hline \multicolumn{2}{|l|}{ Carolina Baeta Neves Duarte Ferreira ................................ 1} \\
\hline \multirow[t]{2}{*}{ Claudia Maria Rodrigues Alves …..................................... 1} & 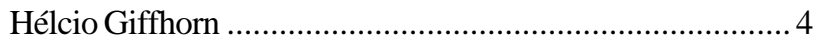 \\
\hline & Henrique Murad.. \\
\hline \multicolumn{2}{|l|}{ Diego Felipe Gaia …….............................................. 1} \\
\hline Djair Brindeiro Filho & Ivan Sergio Joviano Casagrande ……........................ 1 \\
\hline \multicolumn{2}{|l|}{ 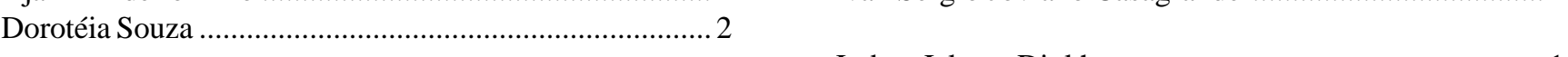 } \\
\hline & Jarbas Jakson Dinkhuysen ............................ \\
\hline 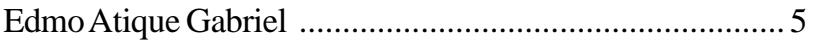 & 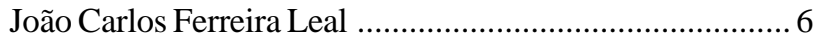 \\
\hline Eduardo Augusto Victor Rocha …................................ 1 & João de Deus e Brito \\
\hline 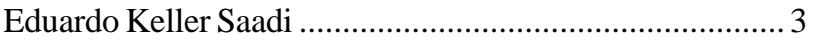 & 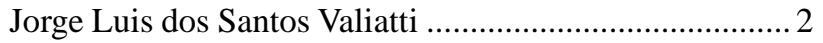 \\
\hline Ektor Correa Vrandecic & José Carlos Dorsa Vieira Pontes ...................................... 1 \\
\hline Elaine Soraya Barbosa de Oliveira Severino ...................... 2 & José Maria Pereira de Godoy ......................................... 1 \\
\hline 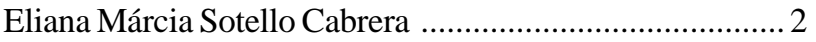 & 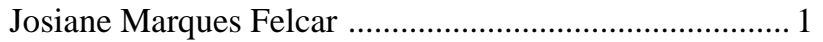 \\
\hline 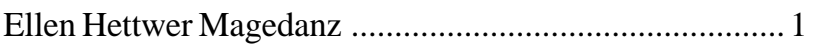 & Juliana Bassalobre Carvalho Borges ................................ 2 \\
\hline \multicolumn{2}{|l|}{ 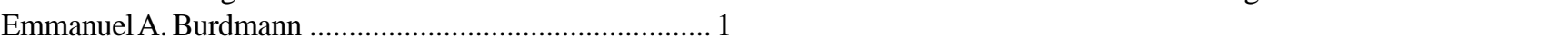 } \\
\hline 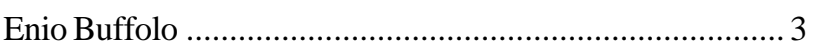 & Karlos Alexandre de Sousa Vilarinho ................................ 5 \\
\hline Fabio Antonio Gaiotto & Lais Helena Carvalho Marino .... \\
\hline 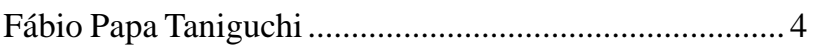 & 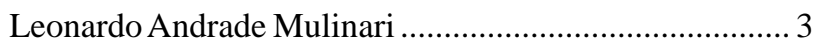 \\
\hline Fausto Miranda Junior & Lilian Beani \\
\hline Fernando Antoniali & 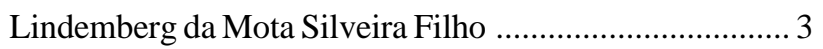 \\
\hline Fernando Platania . & 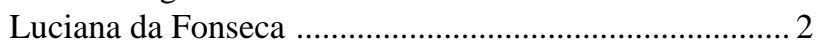 \\
\hline
\end{tabular}


Luciano Cabral Albuquerque ....................................... 3

Luiz Augusto Ferreira Lisboa ....................................... 1

Luiz César Guarita Souza ............................................ 2

Luiz Felipe Pinho Moreira ............................................. 3

Magaly Arrais dos Santos ............................................. 1

Marcela da Cunha Sales .................................................... 2

Marcos Aurélio Barboza de Oliveira ................................ 2

Marcos Rogerio Joaquim ................................................ 1

Marcos Vinícius Pinto e Silva ............................................. 3

Marcus Vinicius Ferraz de Arruda ................................... 1

Maria Cristina de Oliveira Santos Miyazaki .................... 1

Mario Vrandecic .............................................................. 1

Mauricio de Nassau Machado ........................................ 3

Mauro Paes Leme de Sá .................................................. 1

Michel Pereira Cadore ...................................................... 2

Michel Pompeu Barros de Oliveira Sá ............................. 6

Milton Ary Meier ......................................................... 1

Moacir Fernandes de Godoy ........................................ 1

Neuseli Marino Lamari .................................................... 4

Orlando Petrucci .............................................................. 8

Otoni Moreira Gomes ....................................................... 3

Pablo Maria Alberto Pomerantzeff .................................. 3
Paulo Paredes Paulista ................................................. 1

Paulo Roberto Barbosa Evora ........................................ 5

Paulo Roberto Brofman ................................................ 4

Paulo Roberto Lunardi Prates ....................................... 1

Paulo Roberto Prates ............................................... 1

Reinaldo Bestetti .................................................... 4

Reinaldo Wilson Vieira ............................................. 2

Renato Abdala Karam Kalil ........................................... 4

Ricardo de Carvalho Lima ............................................ 1

Ricardo Ribeiro Dias ................................................. 5

Roberto Gomes de Carvalho ........................................... 1

Simone Cavenaghi ......................................................... 1

Solange Guizilini .................................................... 2

Stevan Krieger Martins .......................................... 1

Tomas Salerno ............................................................. 2

Ulisses Alexandre Croti ................................................. 2

Vera Demarchi Aiello ................................................. 1

Vinicius José da Silva Nina ........................................... 2

Walter J Gomes ............................................................ 5 